\title{
A Molecular Networking Strategy: High-Throughput Screening and Chemical Analysis of Brazilian Cerrado Plant Extracts against Cancer Cells
}

\author{
Patrícia C. Cortelo ${ }^{1}\left(\mathbb{D}\right.$, Daniel P. Demarque ${ }^{1}\left(\mathbb{D}\right.$, Renata G. Dusi ${ }^{1,2}{ }^{2}$,orena C. Albernaz ${ }^{1}$, Raimundo Braz-Filho ${ }^{3} \mathbb{C}$, \\ Ekaterina I. Goncharova ${ }^{2,4}$, Heidi R. Bokesch ${ }^{2}$, Kirk R. Gustafson ${ }^{2}{ }^{-}$, John A. Beutler ${ }^{2}$ (i) \\ and Laila S. Espindola 1,2,*(D) \\ 1 Laboratório de Farmacognosia, Universidade de Brasília, Campus Universitário Darcy Ribeiro, \\ Brasília 70910-900, Brazil; pattycar8@gmail.com (P.C.C.); dpdemarque@gmail.com (D.P.D.); \\ renatadusi@hotmail.com (R.G.D.); lalbernaz@unb.br (L.C.A.) \\ 2 Molecular Targets Program, National Cancer Institute, Frederick, MD 21702, USA; \\ katya.goncharova@nih.gov (E.I.G.); heidibokesch@hotmail.com (H.R.B.); gustafki@mail.nih.gov (K.R.G.); \\ beutlerj@mail.nih.gov (J.A.B.) \\ 3 FAPERJ/Departamento de Química, Universidade Federal Rural do Rio de Janeiro, Seropédica, \\ 23897-035 RJ, Brazil and Laboratório de Ciências Químicas, Universidade Estadual do Norte Fluminense, \\ Campos dos Goytacazes, 28013-600 RJ, Brazil; braz@uenf.br \\ check for \\ updates \\ Citation: Cortelo, P.C.; Demarque, \\ 4 Advanced Biomedical Computational Science, Frederick National Laboratory for Cancer Research, \\ Frederick, MD 21702, USA \\ * Correspondence: darvenne@unb.br; Tel.: +55-61-3107-2016
} D.P.; Dusi, R.G.; Albernaz, L.C.; Braz-Filho, R.; Goncharova, E.I.; Bokesch, H.R.; Gustafson, K.R.; Beutler, J.A.; Espindola, L.S. A Molecular Networking Strategy: High-Throughput Screening and Chemical Analysis of Brazilian Cerrado Plant Extracts against Cancer Cells. Cells 2021, 10, 691. https:// doi.org/10.3390/cells10030691

Academic Editors: Franz Hadacek and Petr Karlovsky

Received: 2 February 2021

Accepted: 18 March 2021

Published: 20 March 2021

Publisher's Note: MDPI stays neutral with regard to jurisdictional claims in published maps and institutional affiliations.

Copyright: (c) 2021 by the authors. Licensee MDPI, Basel, Switzerland. This article is an open access article distributed under the terms and conditions of the Creative Commons Attribution (CC BY) license (https:// creativecommons.org/licenses/by/ $4.0 /)$.
Abstract: Plants have historically been a rich source of successful anticancer drugs and chemotherapeutic agents, with research indicating that this trend will continue. In this contribution, we performed high-throughput cytotoxicity screening of 702 extracts from 95 plant species, representing 40 families of the Brazilian Cerrado biome. Activity was investigated against the following cancer cell lines: colon (Colo205 and Km12), renal (A498 and U031), liver (HEP3B and SKHEP), and osteosarcoma (MG63 and MG63.3). Dose-response tests were conducted with 44 of the most active extracts, with 22 demonstrating $\mathrm{IC}_{50}$ values ranging from $<1.3$ to $20 \mu \mathrm{g} / \mathrm{mL}$. A molecular networking strategy was formulated using the Global Natural Product Social Molecular Networking (GNPS) platform to visualize, analyze, and annotate the compounds present in 17 extracts active against NCI-60 cell lines. Significant cytotoxic activity was found for Salacia crassifolia, Salacia elliptica, Simarouba versicolor, Diospyros hispida, Schinus terebinthifolia, Casearia sylvestris var. lingua, Magonia pubescens, and Rapanea guianensis. Molecular networking resulted in the annotation of 27 compounds. This strategy provided an initial overview of a complex and diverse natural product data set, yielded a large amount of chemical information, identified patterns and known compounds, and assisted in defining priorities for further studies.

Keywords: Brazilian Cerrado biome; plant extracts; cancer cell lines; high-throughput screening; NCI-60 cancer cell lines; molecular networking; mass spectrometry

\section{Introduction}

Natural product drug discovery has relied heavily on terrestrial plants, particularly in the anticancer field, with numerous agents of therapeutic importance. According to Newman and Cragg 2020, 41\% of approved antitumor drugs are inspired by natural products; thus, they can be an important source of new structures and can be exploited with synthetic chemistry or synthetic biology [1]. Vinblastine, vincristine, etoposide, and paclitaxel are examples of plant-derived chemotherapeutics currently used in cancer treatment. However, poor solubility in aqueous media, together with significant toxic side effects of many current therapeutics, and limited clinical efficacy, warrant further research 
to discover novel agents with improved characteristics. Analogues or prodrugs of these agents as well as antibody-drug conjugates have been synthesized to increase aqueous solubility and specifically target tumors with reduced off-target effects. The discovery of biologically active structures from natural products remains important to identify potential drug development candidates [1].

The Cerrado is the second largest biome in Brazil, occupying $21 \%$ of the total land area (Figure 1). It is considered a global hotspot for conservation of biodiversity due to its species richness and rapid loss of natural habitats. More than $50 \%$ of the original forest cover has been converted for new uses, mainly croplands and pasturelands. Unfortunately, soil degradation and habitat fragmentation result in pressure on the last large remnants of native vegetation. Besides its biological importance, the Cerrado plays a crucial role in producing and conserving water resources that contribute to three important hydrological basins in Brazil. This contribution is related to the Cerrado soils that are old clayey Oxisols favoring rainwater infiltration. Cerrado vegetation also represents a significant carbon stock in above and below ground biomass. The great diversity of habitats is supported by factors such as distance from watercourses, depth of soil and groundwater, soil composition, frequency of fires, and regional climate. The high number of endemic species and overall species richness make the Cerrado a rich source of potential natural products discoveries and studies [2].

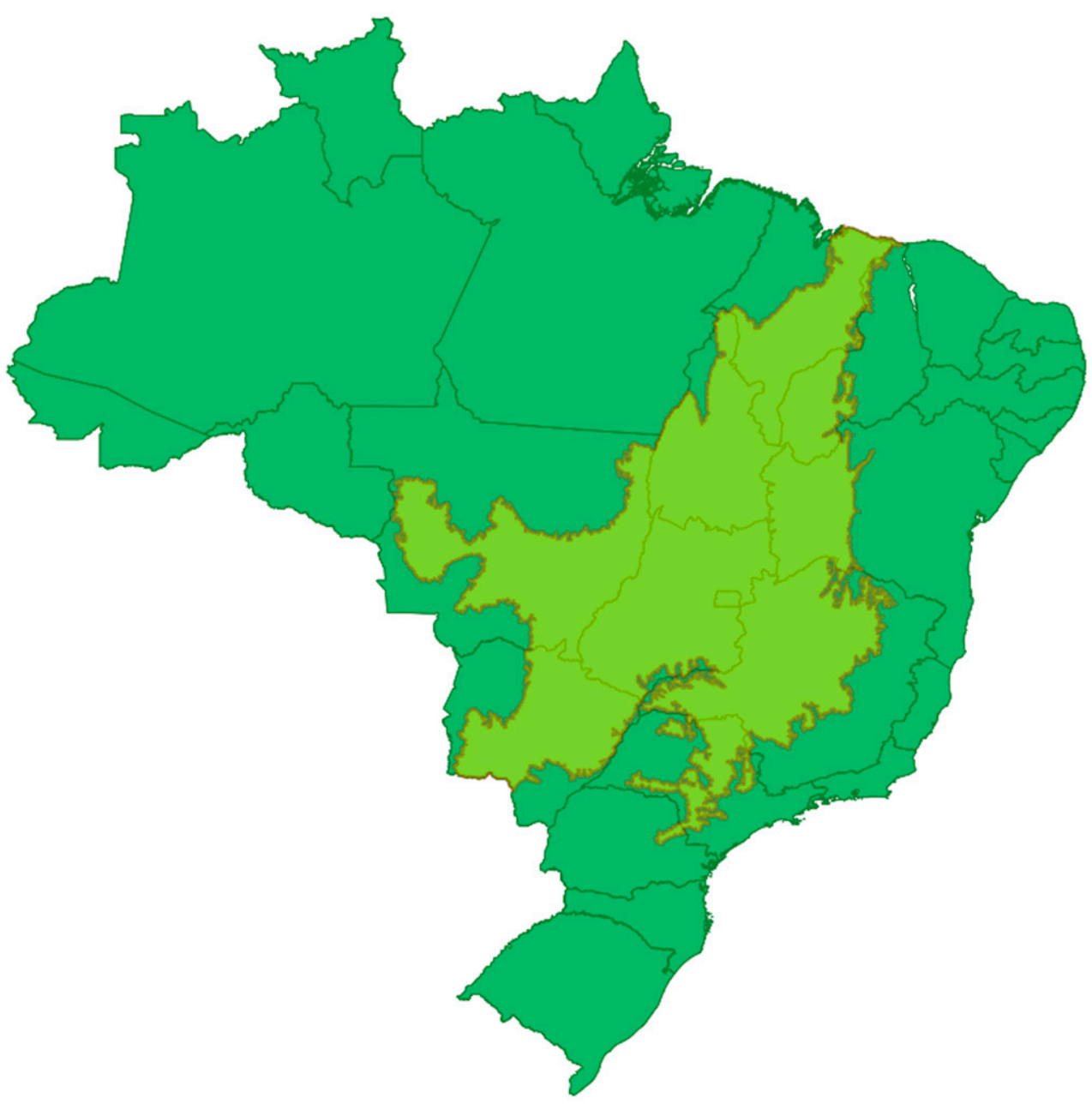

Figure 1. Cerrado delimitation area: light green area in Brazil map. Image from Laboratório de Farmacognosia/UnB adapted from templates available at Project MapBiomas (Project MapBiomasCollection 5 of Brazilian Land Cover \& Use Map Series, accessed on 26 February 2021 through the link: https:/ / mapbiomas.org/mapas-de-referencia?cama_set_language=pt-BR). 
Metabolomic and chemometric tools have played an important role in the discovery of natural bioactive compounds by the exploration and correlation of metabolic profiles, or as dereplication strategies to avoid re-isolation of known compounds [3,4]. One of the most widely used techniques is high-resolution mass spectrometry (HRMS) which can collect data from untargeted (qualitative detection of all measurable metabolites) or targeted (quantification of a specific group of compounds) analyses [4]. In molecular networking, experimental data are analyzed using algorithms to group compounds of the same class according to their mass fragmentation similarities. Comparison with databases (dereplication) in public or in-house libraries enables the annotation of known metabolites. This step can provide an initial overview of vast data sets and helps to guide research priorities [5]. One of the platforms used to perform molecular networking is Global Natural Products Social Molecular Networking (GNPS). This platform consists of an open-access web-based platform available for mass spectrometry raw data analysis (organization, processing and annotation of fragments), storage, and sharing of both raw and processed data, which enables molecular network construction applied to natural products and peptides $[3,4]$.

In the current study, a panel of eight cancer cell lines was utilized, followed by NCI-60 screening and HRMS/MS molecular networking, to evaluate extracts from the Brazilian Cerrado for their cytotoxic activity against tumor cells and explore the associated chemical diversity. The eight cancer cell lines were chosen to include two each from the NCI-60 kidney and colon cancer panels, which respond with different sensitivity to many drugs [6,7], and two each of osteosarcoma [8] and liver cancer cell lines [9] which have rarely been tested with natural products.

Extracts housed at the Laboratório de Farmacognosia (Universidade de Brasília) were evaluated by high-throughput cell screening (HTS) [6-9] and an untargeted mass spectrometry-based molecular networking strategy using the GNPS platform [3]. The NCI-60 cell line screen is a tool employed early in cancer drug discovery and development utilizing a panel of 60 human tumor cell lines representative of a variety of tumor types [10-12]. The assay is used to assess extract or compound activity, and it also provides valuable information regarding potential mechanisms of drug action based on the sensitivity profile of the 60 individual cell lines.

\section{Materials and Methods}

\subsection{Plant Extracts}

Plant material was collected in the Cerrado biome near Brasilia, DF, Brazil. Figure 1 illustrates the original area of Cerrado in Brazil. The Cerrado climate is highly seasonal, marked with dry (May-September) and rainy (October-April) seasons. The different species were collected mainly in the dry season $(74 \%)$, in which the additional stress stimulates Cerrado plants to produce higher metabolite diversity. All individuals were adults and were collected on oxisol or inceptisol soils (Table S1).

These two soil types cover about $85 \%$ of the Federal District in the Cerrado biome's central region. Oxisols are highly weathered and deep soils, with low fertility and a thick, leached horizon of hydrous iron- and aluminum-oxide clays. While oxisols are considered old soils, inceptisols are soils in the early stages of development. This type of soil is less thick and occurs in wavy relief $[13,14]$.

All species were collected by the botanist Professor José Elias de Paula (in memoriam) and voucher specimens were deposited in the Universidade de Brasília (UB/UnB) Herbarium. Plant organs were separated, dried, then pulverized and extracted by maceration with solvents of different polarities. The extract solutions were filtered and concentrated with a rotary evaporator at $35^{\circ} \mathrm{C}$ to yield crude extracts, which were stored at $-20^{\circ} \mathrm{C}$ (Table S1). The Laboratório de Farmacognosia of the Universidade de Brasília is authorized by national authorities to access Brazilian biodiversity under a license granted by CGEN/IBAMA No. 06/2012-Process No. 02000.002272/2006-73. Extract transportation to the United States was authorized under special CGEN/IBAMA exportation license No. 14BR014583/DF. 


\subsection{Antiproliferative Bioassays}

The XTT endpoint assay developed by the Assay Development and Screening Section of the Molecular Targets Program was used for the initial antiproliferative bioassays. The tests were performed as described previously [15]. Extracts were dissolved in DMSO and the 702 extracts were screened at a single concentration of $10 \mu \mathrm{g} / \mathrm{mL}$ against 8 cancer cell lines: Colo205 and Km12 (colon), A498 and U031 (renal), HEP3B and SKHEP (liver), and MG63 and MG63.3 (osteosarcoma). A hit was defined as $>50$ percent inhibition of cell growth. Next, the 44 active extracts were tested in a 5-point dose response format in colon, renal, and osteosarcoma cell lines (Table S2), and the $\mathrm{IC}_{50}$ values determined in duplicate for the 22 most active extracts by regression analysis. Based on these results, 17 extracts were selected for the NCI-60 cell assay. This screen utilizes 60 different human tumor cell lines, representing leukemia, melanoma, and cancers of the lung, colon, brain, ovary, breast, prostate, and kidney. Three endpoints were calculated: (1) $\mathrm{GI}_{50}$, the concentration causing $50 \%$ growth inhibition; (2) TGI, the concentration causing total growth inhibition, and (3) $\mathrm{LC}_{50}$, the concentration causing $50 \%$ lethality of the starting cells $[10,12,16]$.

\subsection{HRESIMS Data Acquisition}

Crude extracts $(25 \mathrm{mg}$ ) were dissolved in methanol and loaded on SPE C18 cartridges (Strata C18-Phenomenex-500 mg). Each cartridge was then eluted with $3 \mathrm{~mL}$ methanol. The resulting fractions were dried in a stream of nitrogen, weighed, and an aliquot resuspended in methanol at $1 \mathrm{mg} / \mathrm{mL}$. The solutions were filtered $(0.22-\mu \mathrm{m}$ filter, Millipore) and a $10-\mu \mathrm{L}$ aliquot injected into a UHPLC-MS/MS system (Bruker Daltonics, Elute pump UHPLC, Elute autosampler UHPLC, Elute DAD) with an ESI-qTOF mass spectrometer (Compact QTOF, Bruker Daltonics). The diode array detector (DAD) was programmed to scan from 200 to $700 \mathrm{~nm}$. The mobile phase used was $\mathrm{MeOH} / \mathrm{H}_{2} \mathrm{O}$, in an exploratory gradient ranging from 5 to $98 \%$ B in A (B: $\mathrm{MeOH} / 0.1 \%$ acetic acid and $\mathrm{A}: \mathrm{H}_{2} \mathrm{O}$ ultrapure $/ 0.1 \%$ acetic acid) for $13 \mathrm{~min}$, the column was stabilized with $5 \% \mathrm{~B}$ for $2 \mathrm{~min}$ at the start and the end of the run, with a flow rate of $0.5 \mathrm{~mL} / \mathrm{min}$. The column used was an Intensity Solo $1.8 \mathrm{C} 18-2$ (Bruker Daltonics), dimensions of $100 \times 2.1 \mathrm{~mm}$, with $1.8-\mu \mathrm{m}$ particles and $100-\AA$ A pore, protected with a pre-column of the same material. The column temperature was set at $40{ }^{\circ} \mathrm{C}$. The samples were maintained at $20^{\circ} \mathrm{C}$ in the autosampler. The data were acquired by Data Analysis software v.4.4 (Bruker Daltonics). The MS adjustment parameters were: desolvation gas (nitrogen) flow of $9 \mathrm{~L} / \mathrm{min}$, at a pressure of 4 bar in the collision cell. The capillary voltage was $4.5 \mathrm{kV}$ and the source temperature was $200{ }^{\circ} \mathrm{C}$. The mass range was $m / z 50-1000$ for both positive and negative ionization modes. The cone voltage and collision energy were $4 \mathrm{eV}$ and $7 \mathrm{eV}$, respectively, for quadrupole and the collision cell. The waiting time was automatically set to $5 \mu$ s. The analysis was performed in Auto_MS.

\subsection{Data Processing and Molecular Networking Construction}

UPLC-MS/MS technology was used to analyze the 17 active extracts assayed in the NCI-60 panel test, in the positive ion mode according to the above conditions. ESI-MS/MS data were converted to .mzXML format using the Bruker data analysis software (v.4.3). The MZmine2 software $[17,18]$ was used for data processing: peak detection and normalization, deconvolution, deisotoping, noise filtering, and gap-filling were set as follows-mass detection at the centroid and chromatogram building used a minimum time span of $0.05 \mathrm{~min}$, a minimum height of 10000, and $m / z$ tolerance of $10 \mathrm{ppm}$. The ADAP (Automated Data Analysis Pipeline) deconvolution algorithm [19] was used (chromatographic threshold $=1 \%$, peak duration range $=0.05-2 \mathrm{~min}$, minimum relative height $=1 \%$, minimum absolute height $=5000$, minimum ratio of peak top/edge $=2$ ). Chromatograms were deisotoped using the isotopic peak grouper algorithm with an $\mathrm{m} / \mathrm{z}$ tolerance of $10 \mathrm{ppm}$ and an RT tolerance of $2 \mathrm{~min}$. Peak alignment was performed using the Join aligner method as follows: $m / z$ tolerance at $10 \mathrm{ppm}$, absolute retention time (RT) tolerance $2 \mathrm{~min}$, absolute RT tolerance after correction of $1 \mathrm{~min}$, and a threshold value of 1 . The peak list was subsequently gap-filled with the peak finder module-intensity tolerance at $5 \%, \mathrm{~m} / z$ tolerance 
at $10 \mathrm{ppm}$, and absolute RT tolerance of $2 \mathrm{~min}$. Molecular networking analysis was performed with an exported MZmine2 $\mathrm{mgf}$ table file into the global natural products social molecular networking (GNPS) online platform using the feature-based molecular networking (FBMN) [20] mode and the following parameters to build networks: clustering the data- precursor ion tolerance was set at $0.1 \mathrm{Da}$; fragment mass tolerance at $0.05 \mathrm{Da}$; cosine score > 0.7; minimum matched fragment ions at 6, and network topK at 10. Finally, the resulting molecular networking was visualized using the Cytoscape software (v.3.7.2) [21]. Annotation was made using the Dictionary of Natural Products (DNP) [22] and Metlin (XCMSonline) databases [23,24].

\section{Results}

A total of 702 Brazilian Cerrado plant extracts from 95 different species, representing 40 families (Table S1), were submitted to high-throughput screening $(10 \mu \mathrm{g} / \mathrm{mL})$ against 8 cancer cell lines. This initial screening against colon (Colo205 and Km12), renal (A498 and U031), liver (HEP3B and SKHEP), and osteosarcoma (MG63 and MG63.3) cells resulted in the selection of 44 active extracts $(6.3 \%)$ for dose-response testing. None of the initial extracts inhibited hepatic cancer cell growth.

The 44 extracts selected were from different plant parts: root wood and root bark (22 extracts, 50\%), stem wood and stem bark (14 extracts, 32\%), leaf (7 extracts, $16 \%$ ), and rhizome (1 extract, $2 \%$ ). Extracts were prepared using different solvents: hexane, ethanol, ethyl acetate, dichloromethane or cyclohexane (Figure 2). Of the 702 extracts, 207 (31\%) were from the root and $270(39 \%)$ were from the stem. While 44 extracts $(6.3 \%$ of the extract bank) were active, a higher percentage $(10 \%)$ of the bank's root extracts were cytotoxic (21/207 root extracts), an indication that the root may constitute a chemically rich organ in Cerrado plants, possessing defensive metabolites with inhibitory properties, including cancer cell line growth inhibition. In previous screening studies involving the same extract library against protozoa and fungi, the root and stem extracts proved the most promising, primarily root and stem bark extracts [25].

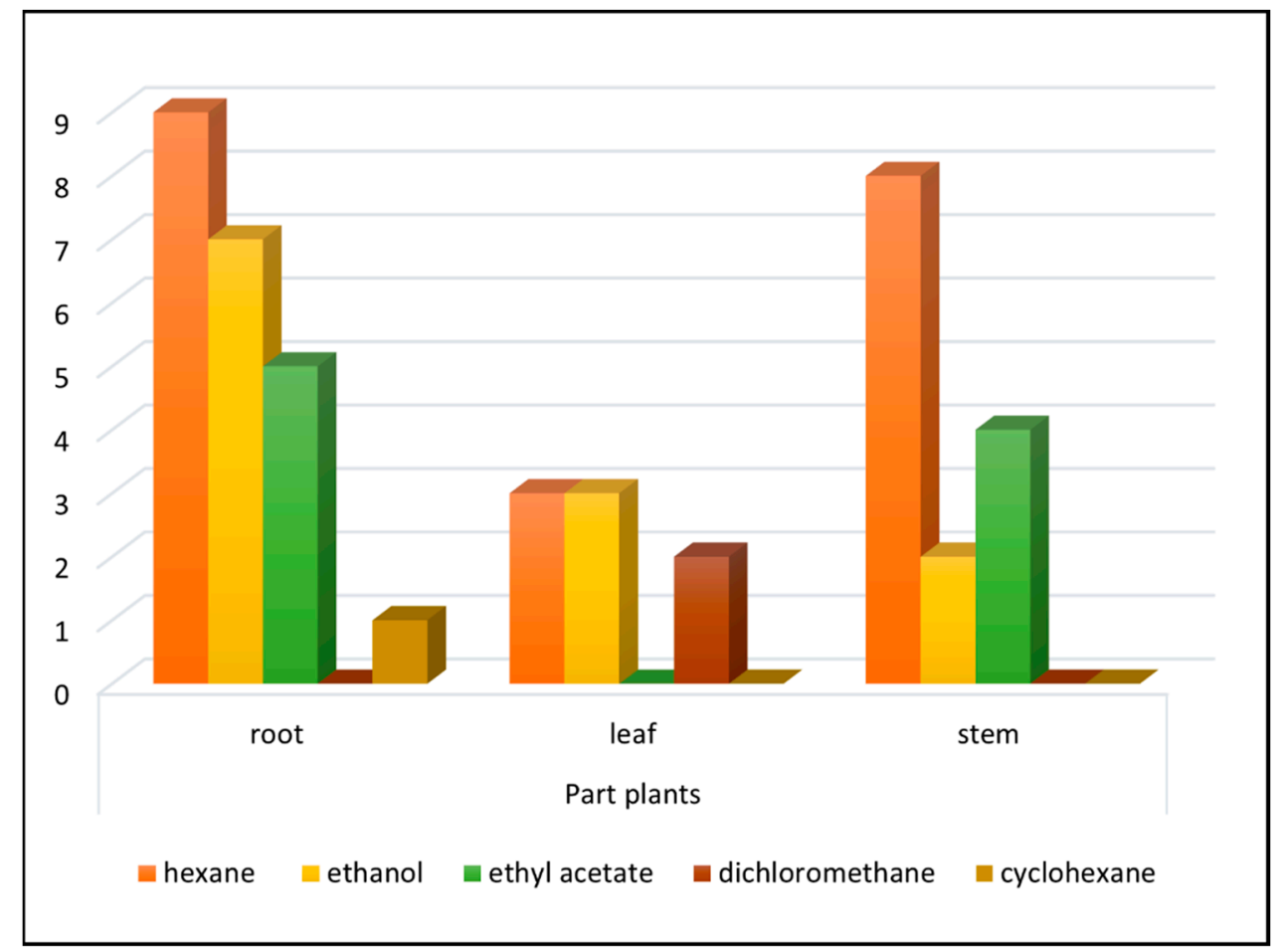

Figure 2. Plant parts and extraction solvents of the 44 active extracts selected for dose-response determination. 


\subsection{High-Throughput Screening}

Dose-response curves (of 44 extracts tested) were generated and $\mathrm{IC}_{50}$ values of the 22 most active extracts ranged from 1.3 to $20 \mu \mathrm{g} / \mathrm{mL}$ against 6 cell lines (Table 1). The Z-factors for plates used in initial screening and secondary dose response testing are described in Table S2. The hexane extract of Salacia crassifolia (Celastraceae) root wood was cytotoxic against the KM12 colon cancer cell line $\left(\mathrm{IC}_{50} 1.7 \mu \mathrm{g} / \mathrm{mL}\right)$ and demonstrated no activity against the Colo205 colon cancer cells. Similarly, it was active against the A498 renal cancer cell line $\left(\mathrm{IC}_{50} 1.6 \mu \mathrm{g} / \mathrm{mL}\right.$ ) but showed no activity against renal cancer U031 cells. The Salacia elliptica root wood EtOAc extract was active against all 6 cell lines ( $\mathrm{IC}_{50}$ 2.0 to $7.1 \mu \mathrm{g} / \mathrm{mL}$ ), with the highest activity against U031 of the extracts tested.

Table 1. Brazilian Cerrado plant extract activity against cancer cell lines ( $\mathrm{IC}_{50} \mathrm{\mu g} / \mathrm{mL}$ ) and NCI-60 sensitivity (potency in $\mu \mathrm{g} / \mathrm{mL})$.

\begin{tabular}{|c|c|c|c|c|c|c|c|c|c|c|}
\hline \multirow{3}{*}{$\begin{array}{c}\text { Plant } \\
\text { Species/Plant } \\
\text { Organ (Solvent) * }\end{array}$} & \multirow[b]{3}{*}{$\begin{array}{c}\text { Codes } \\
\text { BR/NCI60 }\end{array}$} & \multicolumn{6}{|c|}{$\mathrm{IC}_{50}(\mu \mathrm{g} / \mathrm{mL})$} & \multirow{2}{*}{\multicolumn{3}{|c|}{$\begin{array}{l}\text { NCI-60 Sensitivity } \\
\text { Potency }(\mu \mathrm{g} / \mathrm{mL})\end{array}$}} \\
\hline & & \multicolumn{2}{|c|}{ Colon } & \multicolumn{2}{|c|}{ Renal } & \multicolumn{2}{|c|}{ Osteosarcoma } & & & \\
\hline & & Colo205 & KM12 & A498 & U031 & MG63 & MG 63.3 & $\begin{array}{c}\text { Mean } \\
\text { GI }_{50} / \text { Most } \\
\text { Sensitive Cell } \\
\text { Line }\end{array}$ & $\begin{array}{c}\text { Mean } \\
\text { TGI }\end{array}$ & $\begin{array}{l}\text { Mean } \\
\text { LC }_{50}\end{array}$ \\
\hline $\begin{array}{c}\text { Peschiera affinis } \\
\text { SB (h) }\end{array}$ & BR 075 & 17.8 & 19.1 & $>20$ & $>20$ & $>20$ & $>20$ & - & - & - \\
\hline $\begin{array}{c}\text { Cybistax } \\
\text { antisyphilitica } \\
\text { SB (h) }\end{array}$ & $\begin{array}{c}\text { BR } 125 \\
\text { N192795 }\end{array}$ & - & - & - & - & - & - & 85 & $>100$ & $>100$ \\
\hline $\begin{array}{c}\text { Tabebuia caraiba } \\
\text { L (h) }\end{array}$ & BR 139 & $>20$ & 18.9 & $>20$ & $>20$ & $>20$ & $>20$ & - & - & - \\
\hline $\begin{array}{c}\text { Casearia sylvestris } \\
\text { var. lingua } \\
\text { SW }(\mathrm{h})\end{array}$ & $\begin{array}{c}\text { BR } 177 \\
\text { N192825 }\end{array}$ & 4.6 & 4.5 & 3.6 & 2.7 & 3.9 & 5.7 & $\begin{array}{c}11 / 2.5 \\
\text { NCI-H522 }\end{array}$ & 28 & 76 \\
\hline $\begin{array}{c}\text { Cupania vernalis } \\
\mathrm{L}(\mathrm{h})\end{array}$ & $\begin{array}{c}\text { BR } 193 \\
\text { N192827 }\end{array}$ & 12.3 & 10 & 18.7 & 7.6 & 13.8 & 15 & 43 & 140 & 490 \\
\hline $\begin{array}{c}\text { Magonia pubescens } \\
\text { R (e) }\end{array}$ & BR 197 & 4.2 & 7.2 & 3.6 & 3.9 & 14.5 & 14.5 & - & - & - \\
\hline $\begin{array}{c}\text { Magonia pubescens } \\
\text { RW (e) }\end{array}$ & $\begin{array}{l}\text { BR } 204 \\
\text { N192797 }\end{array}$ & 3.5 & 5.5 & 2.9 & 3.8 & 15.4 & 15.6 & $15 / 0.3 S R$ & 34 & 81 \\
\hline $\begin{array}{l}\text { Simarouba } \\
\text { versicolor } \\
\text { RB (e) }\end{array}$ & $\begin{array}{c}\text { BR } 254 \\
\text { N192829 }\end{array}$ & 6.8 & $>20$ & 3.7 & 5 & $<1.3$ & $<1.3$ & $\begin{array}{c}4.9 / 0.7 \\
\text { NCI-H522 }\end{array}$ & 29 & 620 \\
\hline $\begin{array}{c}\text { Simaba suffruticosa } \\
\text { L (a) }\end{array}$ & BR 261 & 16.8 & 1.6 & $>20$ & 17.6 & 2.2 & 4.9 & - & - & - \\
\hline $\begin{array}{c}\text { Kielmeyera coriacea } \\
\text { SW (h) }\end{array}$ & $\begin{array}{c}\text { BR } 331 \\
\text { N192831 }\end{array}$ & 11.9 & 4.9 & 15.9 & 14.9 & $>20$ & $>20$ & 56 & 210 & 600 \\
\hline $\begin{array}{c}\text { Byrsonima crassa } \\
\text { RB (h) }\end{array}$ & $\begin{array}{c}\text { BR } 411 \\
\text { N192833 }\end{array}$ & 15 & 15 & $>20$ & $>20$ & $>20$ & $>20$ & 220 & 530 & 910 \\
\hline $\begin{array}{l}\text { Schinus } \\
\text { terebinthifolia } \\
\text { L (d) }\end{array}$ & $\begin{array}{c}\text { BR } 436 \\
\text { N192835 }\end{array}$ & 12.4 & 6.9 & 16.2 & 7.8 & 3.4 & 14.8 & $\begin{array}{c}100 / 0.9 \\
\text { NCI-H522 }\end{array}$ & 87 & 360 \\
\hline $\begin{array}{l}\text { Enterolobium } \\
\text { gummiferum } \\
\text { RW (e) }\end{array}$ & BR 467 & 10.5 & 10.9 & $<1.3$ & 4.6 & 12.6 & 14.9 & - & - & - \\
\hline $\begin{array}{l}\text { Enterolobium } \\
\text { gummiferum } \\
\text { SB (h) }\end{array}$ & $\begin{array}{l}\text { BR } 469 \\
\text { N192837 }\end{array}$ & 2.2 & 4.9 & $>20$ & $>20$ & $>20$ & $>20$ & 180 & 710 & 980 \\
\hline $\begin{array}{l}\text { Plathymenia } \\
\text { reticulata } \\
\text { RW (h) }\end{array}$ & $\begin{array}{c}\text { BR } 489 \\
\text { N192839 }\end{array}$ & 8.9 & 6.7 & 12.4 & 4.9 & $>20$ & $>20$ & 60 & 330 & 680 \\
\hline $\begin{array}{c}\text { Diospyros hispida } \\
\text { R (a) }\end{array}$ & $\begin{array}{c}\text { BR } 501 \\
\text { N192799 }\end{array}$ & - & - & - & - & - & - & $\begin{array}{c}7 / 0.7 \\
\text { NCI-H522 }\end{array}$ & 30 & 81 \\
\hline
\end{tabular}


Table 1. Cont

\begin{tabular}{|c|c|c|c|c|c|c|c|c|c|c|}
\hline \multirow{3}{*}{$\begin{array}{c}\text { Plant } \\
\text { Species/Plant } \\
\text { Organ (Solvent) * }\end{array}$} & \multirow[b]{3}{*}{$\begin{array}{c}\text { Codes } \\
\text { BR/NCI60 }\end{array}$} & \multicolumn{6}{|c|}{$\mathrm{IC}_{50}(\mu \mathrm{g} / \mathrm{mL})$} & \multirow{2}{*}{\multicolumn{3}{|c|}{$\begin{array}{l}\text { NCI-60 Sensitivity } \\
\text { Potency }(\mu \mathrm{g} / \mathrm{mL})\end{array}$}} \\
\hline & & \multicolumn{2}{|c|}{ Colon } & \multicolumn{2}{|c|}{ Renal } & \multicolumn{2}{|c|}{ Osteosarcoma } & & & \\
\hline & & Colo205 & KM12 & A498 & U031 & MG63 & MG 63.3 & $\begin{array}{c}\text { Mean } \\
\text { GI }_{50} / \text { Most } \\
\text { Sensitive Cell } \\
\text { Line }\end{array}$ & $\begin{array}{c}\text { Mean } \\
\text { TGI }\end{array}$ & $\begin{array}{l}\text { Mean } \\
\mathrm{LC}_{50}\end{array}$ \\
\hline $\begin{array}{l}\text { Maprounea } \\
\text { guianensis } \\
\text { RB (a) }\end{array}$ & BR 536 & 8.1 & 9.9 & 6.7 & $>20$ & 9.6 & 8.5 & - & - & - \\
\hline $\begin{array}{c}\text { Psidium } \\
\text { laruotteanum } \\
\text { SB (h) }\end{array}$ & $\begin{array}{c}\text { BR } 549 \\
\text { N192841 }\end{array}$ & - & - & - & - & - & - & 87 & 340 & 830 \\
\hline $\begin{array}{c}\text { Andira humilis } \\
\text { SB (e) }\end{array}$ & BR 587 & $>20$ & 18.6 & $>20$ & $>20$ & $>20$ & $>20$ & - & - & - \\
\hline $\begin{array}{c}\text { Rapanea guianensis } \\
\text { SW }(\mathrm{h})\end{array}$ & BR 624 & 5.3 & 3.2 & 4.3 & 4.4 & 17.8 & 14.9 & - & - & - \\
\hline $\begin{array}{c}\text { Rapanea guianensis } \\
\text { RW (e) }\end{array}$ & $\begin{array}{c}\text { BR } 627 \\
\text { N192801 }\end{array}$ & 3.5 & 4.0 & 2.7 & 3.7 & 14.6 & 14 & $\begin{array}{l}16 / 2.4 \\
\text { SR }\end{array}$ & 361 & 81 \\
\hline $\begin{array}{c}\text { Salacia crassifolia } \\
\text { RW (h) }\end{array}$ & $\begin{array}{c}\text { BR } 640 \\
\text { N192803 }\end{array}$ & $>20$ & 1.7 & 1.6 & $>20$ & 3.7 & 5.7 & $\begin{array}{c}0.3 / 0.1 \\
\text { HCT-15 }\end{array}$ & 1.0 & 5.0 \\
\hline $\begin{array}{c}\text { Salacia elliptica } \\
\text { RW (a) }\end{array}$ & $\begin{array}{c}\text { BR } 652 \\
\text { N192805 }\end{array}$ & 3.6 & 3.4 & 3.5 & 2.0 & 5.1 & 7.1 & $\begin{array}{c}2.0 / 0.7 \\
\text { MOLT-4 }\end{array}$ & 5.1 & 20 \\
\hline $\begin{array}{c}\text { Lippia rotundifolia } \\
\text { SW (a) }\end{array}$ & $\begin{array}{c}\text { BR } 660 \\
\text { N192843 }\end{array}$ & 12.3 & 9.9 & $>20$ & $>20$ & $>20$ & $>20$ & - & - & - \\
\hline $\begin{array}{c}\text { Connarus } \\
\text { suberosus } \\
\text { RW (a) }\end{array}$ & $\begin{array}{c}\text { BR } 693 \\
\text { N192845 }\end{array}$ & 5.7 & 4.5 & 4.7 & 7.7 & 17.6 & 19 & 44 & 200 & 830 \\
\hline
\end{tabular}

$\mathrm{IC}_{50}$ : inhibitory concentration (50\%) * Plant organ: L-leaf; SB-stem bark; SW-stem wood; R-root (wood + bark); RW-root wood; RB-root bark; Rz-rhizome. *Extraction solvent: h-hexane; e-ethanol; a-ethyl acetate. $\mathrm{GI}_{50}$ : the concentration causing $50 \%$ growth inhibition; TGI: the concentration causing total growth inhibition; $\mathrm{LC}_{50}$ : the concentration causing $50 \%$ lethality of the starting cells.

The ethanol extract of Simarouba versicolor (Simaroubaceae) root bark was the most cytotoxic extract against both osteosarcoma cell lines, MG63 and MG63.3, with $\mathrm{IC}_{50}$ values $<1.3 \mu \mathrm{g} / \mathrm{mL}$. The Schinus terebinthifolia (Anacardiaceae) leaf dichloromethane extract was also active against MG63 ( $\mathrm{IC}_{50} 3.4 \mu \mathrm{g} / \mathrm{mL}$ ). Casearia sylvestris var. lingua (Salicaceae) stem wood hexane extract was cytotoxic against the 6 cell lines ( $\mathrm{IC}_{50} 2.7$ to $5.4 \mu \mathrm{g} / \mathrm{mL}$ ). The Enterolobium gummiferum (Fabaceae) root wood ethanol extract was active against both renal cell lines, with higher activity against A498 cells $\left(\mathrm{IC}_{50}<1.3 \mu \mathrm{g} / \mathrm{mL}\right)$. For this same species, the stem bark hexane extract was selective for colon cancer cells Colo205 and KM12, with $\mathrm{IC}_{50} 2.2$ and $4.9 \mu \mathrm{g} / \mathrm{mL}$, respectively. Two of the Rapanea guianensis (Primulaceae) extracts (stem wood hexane and root wood ethanol) showed activity against the colon and renal cells tested, with $\mathrm{IC}_{50}$ values between 2.7 and $5.3 \mu \mathrm{g} / \mathrm{mL}$ (Table 1).

Based on the selective cytotoxicity results, we submitted the 17 most active extracts to NCI-60 cell one-dose and then five-dose screening (Figures S5-S55). The strongest activity was found for the S. crassifolia root wood hexane extract (BR 640; N192803), presenting a mean $\mathrm{GI}_{50} 0.3 \mu \mathrm{g} / \mathrm{mL}$ for all 60 cell lines, with the HCT- 15 colon cancer cell line the most sensitive $\left(\mathrm{GI}_{50} 0.1 \mu \mathrm{g} / \mathrm{mL}\right)$ (Table 1). Another Salacia species extract, S. elliptica root wood EtOAc (BR 652; N192805), demonstrated a mean GI $502.0 \mu \mathrm{g} / \mathrm{mL}$ and was most potent against the leukemia MOLT-4 cell line $\left(\mathrm{GI}_{50} 0.7 \mu \mathrm{g} / \mathrm{mL}\right.$ ) (Table 1). Simarouba versicolor root bark ethanol (BR 254; N192829) and Diospyros hispida (Ebenaceae) root (wood and bark) EtOAc (BR 501; N192799) extracts, displayed similar results against the non-small cell lung NCI-H522 cell line ( $\mathrm{GI}_{50} 0.7 \mu \mathrm{g} / \mathrm{mL}$ ) (Table 1 ). This cell line was also sensitive to the $S$. terebinthifolia leaf dichloromethane (BR 436; N192835) and C. sylvestris var. lingua stem wood hexane (BR 177; N192825) extracts, with $\mathrm{GI}_{50}$ values of $0.9 \mu \mathrm{g} / \mathrm{mL}$ and $2.5 \mu \mathrm{g} / \mathrm{mL}$, respectively (Table 1). Both the Magonia pubescens (Sapindaceae) (BR 204; N192797) and 
Rapanea guianensis (BR 627; N192801) root wood ethanol extracts were primarily active against the leukemia SR cell line, with respective GI $_{50}$ values of $0.3 \mu \mathrm{g} / \mathrm{mL}$ and $2.4 \mu \mathrm{g} / \mathrm{mL}$ (Table 1).

\subsection{Molecular Networking}

Analysis of the 17 most active extracts using a MS/MS dereplication approach resulted in the construction of the molecular network. The LC-MS/MS data were converted to .mzXML files, preprocessed using the MZmine2 $[17,18]$ software in order to align and detect the most relevant information, together with the most intense chromatographic peaks. The resulting .cvs table was uploaded to the GNPS platform and organized according to fragmentation pattern profile similarities. This process improved data visualization by generating a network with clusters corresponding to different compound classes. Clusters are groups of nodes (plotted here as pie charts representing compound percentages in each of the 17 extracts) and the connecting lines constitute edges (cosine values that represent related patterns between nodes). In this study, the total molecular networking (MN), visualized in the Supplementary Material as Figure S1, consists of 47 clusters and 279 selfloops. It was possible to annotate 25 compounds. An additional 2 compounds were annotated using public databases-Dictionary of Natural Products (DNP) [22] and Metlin [23,24]. The annotated compounds, 27 in total, are listed in Table 2.

Table 2. Compounds annotated by molecular networking of 17 Cerrado plant crude extracts.

\begin{tabular}{|c|c|c|c|}
\hline Compound Name & Molecular Formula & $m / z$ & Rt (min) \\
\hline oleanolic acid* & $\mathrm{C}_{30} \mathrm{H}_{48} \mathrm{O}_{3}$ & 439.3567 & 9.7 \\
\hline ursolic acid* & $\mathrm{C}_{30} \mathrm{H}_{48} \mathrm{O}_{3}$ & 439.3567 & 9.7 \\
\hline $3 \alpha$-cyclopenta $[\alpha]$ chrysene- $3 \alpha$-carboxylic acid & $\mathrm{C}_{29} \mathrm{H}_{46} \mathrm{O}_{4}$ & 441.3367 & 7.5 \\
\hline platanic acid & $\mathrm{C}_{29} \mathrm{H}_{46} \mathrm{O}_{4}$ & 459.346 & 7.6 \\
\hline linoleic acid & $\mathrm{C}_{18} \mathrm{H}_{32} \mathrm{O}_{2}$ & 281.2476 & 8.4 \\
\hline linoleic acid ethyl ester & $\mathrm{C}_{20} \mathrm{H}_{36} \mathrm{O}_{3}$ & 309.2787 & 10.0 \\
\hline linolenic acid ethyl ester & $\mathrm{C}_{20} \mathrm{H}_{34} \mathrm{O}_{2}$ & 307.2635 & 9.6 \\
\hline 13-keto-9Z,11E-octadecadienoic acid & $\mathrm{C}_{18} \mathrm{H}_{30} \mathrm{O}_{3}$ & 295.2272 & 8.4 \\
\hline 9-oxo-10E,12Z-octadecadienoic acid & $\mathrm{C}_{18} \mathrm{H}_{30} \mathrm{O}_{3}$ & 295.2269 & 8.5 \\
\hline stearidonic acid & $\mathrm{C}_{18} \mathrm{H}_{28} \mathrm{O}_{2}$ & 277.2166 & 8.3 \\
\hline 9(10)-epoxy-12Z-octadecenoic acid & $\mathrm{C}_{18} \mathrm{H}_{32} \mathrm{O}_{3}$ & 279.2322 & 8.1 \\
\hline 9S,13R-12-oxophytodienoic acid & $\mathrm{C}_{18} \mathrm{H}_{28} \mathrm{O}_{3}$ & 293.2098 & 5.3 \\
\hline pristimerin & $\mathrm{C}_{30} \mathrm{H}_{40} \mathrm{O}_{4}$ & 465.3012 & 10.3 \\
\hline tingenone & $\mathrm{C}_{28} \mathrm{H}_{36} \mathrm{O}_{3}$ & 421.2745 & 8.6 \\
\hline 20-oxo-20,21-seco-tingen-21-oic acid & $\mathrm{C}_{28} \mathrm{H}_{36} \mathrm{O}_{5}$ & 453.263 & 5.9 \\
\hline$(-)$-catechin gallate & $\mathrm{C}_{22} \mathrm{H}_{18} \mathrm{O}_{10}$ & 443.0972 & 3.7 \\
\hline epigallocatechin gallate & $\mathrm{C}_{22} \mathrm{H}_{17} \mathrm{O}_{11}$ & 459.0944 & 3.2 \\
\hline luteolin $3^{\prime}, 4^{\prime}$-di-O-beta-D-glucopyranoside & $\mathrm{C}_{27} \mathrm{H}_{29} \mathrm{O}_{16}$ & 628.1959 & 10.7 \\
\hline 13-docosenamide & $\mathrm{C}_{22} \mathrm{H}_{43} \mathrm{NO}_{3}$ & 338.342 & 11.7 \\
\hline 9-octadecenamide & $\mathrm{C}_{18} \mathrm{H}_{35} \mathrm{NO}$ & 282.2793 & 10.1 \\
\hline N-phenyl-1-naphthylamine & $\mathrm{C}_{16} \mathrm{H}_{13} \mathrm{~N}$ & 220.1124 & 8.2 \\
\hline $\begin{array}{l}\text { (2R,3S,4S,5R,6S)-2-[[(2S,3R,4R)-3,4-dihydroxy-4- } \\
\text { (hydroxymetil)oxolan-2-yl]oxymetil]-6-(3,4,5- } \\
\text { trimethoxyphenoxy)oxane-3,4,5-triol }\end{array}$ & $\mathrm{C}_{20} \mathrm{H}_{30} \mathrm{O}_{13}$ & 496.1988 & 3.0 \\
\hline $\begin{array}{c}\text { (3R,5R,6R,7S,9S,10R,13R,17R)-17-((R)-5-ethoxy-5-oxypentan-2-yl)- } \\
\text { 10,13-dimethylhexadecahydro-1H-cyclopenta[a]phenanthreno- } \\
\text { 3,6,7-triyl } \\
\text { triacetate }\end{array}$ & $\mathrm{C}_{32} \mathrm{H}_{50} \mathrm{O}$ & 585.3039 & 9.0 \\
\hline 1-linoleoilglycerol & $\mathrm{C}_{16} \mathrm{H}_{36} \mathrm{O}_{3}$ & 355.282 & 7.9 \\
\hline hesperidine & $\mathrm{C}_{28} \mathrm{H}_{34} \mathrm{O}_{15}$ & 611.498 & 4.1 \\
\hline palmitamide & $\mathrm{C}_{20} \mathrm{H}_{41} \mathrm{NO}$ & 256.2639 & 10.0 \\
\hline $\begin{array}{l}\text { 4-(2,6,6-trimethyl-4-oxo-2-ciclohexen-1-yl)-2-butanyl } \\
\text { beta-D-glucopyranoside }\end{array}$ & $\mathrm{C}_{19} \mathrm{H}_{32} \mathrm{O}_{7}$ & 373.2192 & 4.2 \\
\hline
\end{tabular}


To better visualize and analyze the data collected from the 17 extracts selected, we performed a molecular networking workflow associated with high-throughput cancer screening. Detailed inspection of one main cluster (Figure 3) showed that the metabolites were grouped into three sub-networks (G): G1-triterpenes; G2-oleanolic/ursolic acids and derivatives; and G3-essential fatty acids-linoleic and linolenic acids and derivatives interconnected by common biosynthetic routes (biosynthesis of fatty acids, unsaturated fatty acids, elongation and reduction of the fatty acid chain). The metabolites here are present in all 17 plant extracts, except for triterpenes that are mainly detected in Salacia species.

G2

oleanolic and ursolic acids and derivatives

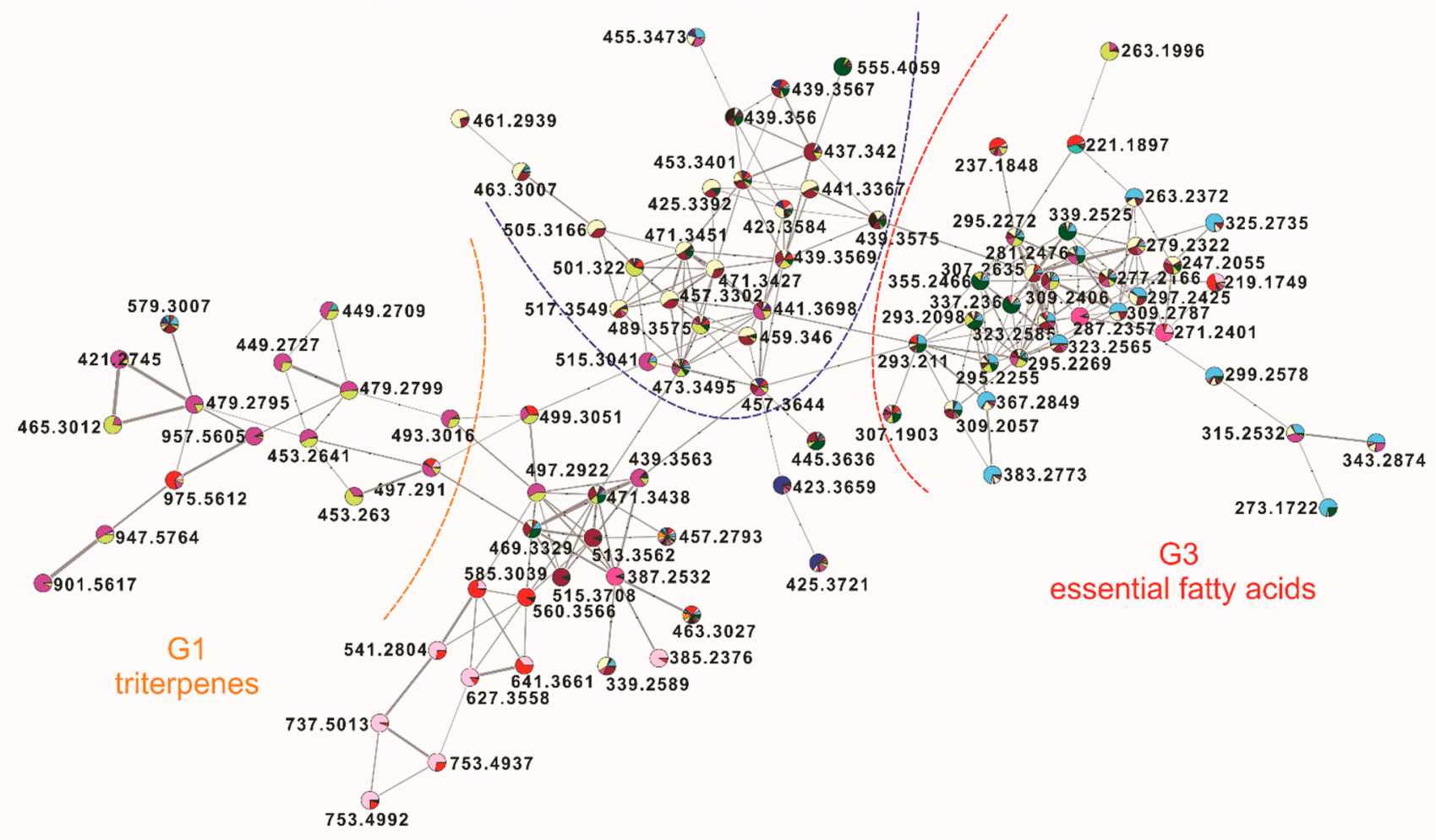

Legend:

- Cybistax antisyphilitica

- Casearia sylvestris var. lingua

- Cupania vernalis

- Magonia pubescens

- Connarus suberosus
- Simarouba versicolor

- Kielmeyera coriacea

- Byrsonima crassa

- Schinus terebinthifolia
Enterolobium gummiferum

- Plathymenia reticulata

- Diospyros hispida

- Psidium laruotteanum.
- Rapanea guianensis

- Salacia crassifolia

- Salacia elliptica

- Lippia rotundifolia

Figure 3. Main cluster-3 sub-networks: G1 triterpenes; G2 oleanolic and ursolic acids and derivatives; G3 essential fatty acids-linoleic and linolenic acids and derivatives.

In the $\mathrm{G} 2$ sub-network, oleanolic acid $\left[\mathrm{M}+\mathrm{H}-\mathrm{H}_{2} \mathrm{O}\right]^{+} \mathrm{m} / z$ 439.3567; $3 \alpha$-cyclopenta $[\alpha]$ chrysene- $3 \alpha$-carboxylic acid $\left[\mathrm{M}+\mathrm{H}-\mathrm{H}_{2} \mathrm{O}\right]^{+} \mathrm{m} / z$ 441.3367, and platanic acid $[\mathrm{M}+\mathrm{H}]^{+}$ $\mathrm{m} / \mathrm{z} 459.346$ were annotated. Oleanolic and ursolic acids are pentacyclic triterpene isomers, differing in the methyl group position in the E ring [26]. Both acids are used in Chinese medicine as hepatoprotectives, with oleanolic acid also possessing anti-tumor activity [27,28], and ursolic acid possessing anti-HIV, anti-inflammatory, antiulcer, hypolipidemic, and antiatherosclerotic activities [29]. Platanic acid was isolated primarily from Syzygium claviflorum leaves that exhibit anti-HIV activity, with studies of its derivatives suggesting antitumoral activity [30-32]. We observed that the G3 sub-network contains a conjugated linoleic acid $[\mathrm{M}+\mathrm{H}]^{+} \mathrm{m} / z 281.2476$ and derivatives: ethyl-linoleic ester $[\mathrm{M}+\mathrm{H}]^{+}$ $\mathrm{m} / \mathrm{z}$ 309.2787; ethyl-linolenic ester [M + H] ${ }^{+} \mathrm{m} / \mathrm{z}$ 307.2635; 9-oxo-10E,12Z-octadecadienoic acid $[\mathrm{M}+\mathrm{H}]^{+} \mathrm{m} / \mathrm{z}$ 295.2269; 13-keto-9Z,11E-octadecadienoic acid $[\mathrm{M}+\mathrm{H}]^{+} \mathrm{m} / \mathrm{z}$ 295.2272; 
stearidonic acid $[\mathrm{M}+\mathrm{H}]^{+} m / z$ 277.2166; 9(10)-epoxy-12Z-octadecenoic acid $\left[\mathrm{M}+\mathrm{H}-\mathrm{H}_{2} \mathrm{O}\right]^{+}$ $m / z$ 279.2322, and 9S,13R-12-oxophytodienoic acid $[\mathrm{M}+\mathrm{H}]^{+} \mathrm{m} / \mathrm{z} 293.2098$ (Table 2). These essential fatty acids are produced exclusively by plants [33]. Recent research suggests that a diet enriched with linoleic acid is related to a reduction in the incidence of cancer [32,33]. Linolenic and linoleic acids may act via different mechanisms to inhibit the development of some types of cancer, especially breast cancer [33-35]. The G1 sub-network contains: pristimerin $[\mathrm{M}+\mathrm{H}]^{+} \mathrm{m} / \mathrm{z} 465.3012$; tingenone $[\mathrm{M}+\mathrm{H}]^{+} \mathrm{m} / \mathrm{z}$ 421.2745; 20-oxo20,21-seco-tingen-21-oic acid $[\mathrm{M}+\mathrm{H}]^{+} \mathrm{m} / \mathrm{z} 453.263$, and other unidentified derivatives in the database. The common fragment $(m / z$ 201.0609) suggests that these unidentified derivatives resulted from fragmentation involving carbocation formation, followed by displacement of a methyl and pericyclic rearrangement. They are predominantly present in S. crassifolia and S. elliptica, with reported activity against cancer cell lines [36,37].

In minor clusters (Figure S3, purple highlighted circle), it was possible to annotate a catechin derivative cluster: catechin gallate $[\mathrm{M}+\mathrm{H}]^{+} \mathrm{m} / z 443.0972$ and epigallocatechin gallate $[\mathrm{M}+\mathrm{H}]^{+} m / z$ 459.0944. These catechin-derived flavonoids have been studied for their properties of inhibiting pancreatic cancer with promising results $[38,39]$. In another cluster (Figure S3, orange highlighted circle), luteolin $3^{\prime}, 4^{\prime}$-di-O-beta-D-glucopyranoside, a glycosylated flavone with $\left[\mathrm{M}+\mathrm{NH}_{4}\right]^{+} m / z 628.1959$ was annotated [40]. Although it was not possible to identify other compounds here, the fragment ions obtained showed that they were glycosylated flavones probably derived from the aforementioned annotated flavone.

\section{Discussion}

Cancer remains a complex and challenging disease that is often fatal [41]. Natural products constitute a high percentage of chemotherapeutic and chemoprevention drugs, which may be used in isolation or as part of a combined cancer treatment strategy [41]. The cytotoxicity data in this study supports the Brazilian Cerrado biome as a rich source of potential chemotherapeutic agents.

Initial screening of 702 plant extracts against 8 different cell lines resulted in the selection of 44 active extracts for dose-response determination. This data, together with the $\mathrm{IC}_{50}$ value determination of 22 of these extracts, led to NCI-60 cell screening of the 17 most active extracts, corresponding to $2.4 \%$ of the Brazilian Cerrado biome extract bank. This test has been used for the last three decades to select chemicals and natural product extracts with the ability to inhibit the growth of, or kill, cancer cells [10,11].

Some of these 17 extracts were previously investigated against other biological targets such as: (i) protozoa-S. terebinthifolia and D. hispida (Plasmodium falciparum) [42], C. sylvestris var. lingua (Trypanosoma cruzi and Leishmania donovani) [43], C. vernalis (L. donovani) [43] and C. suberosus (L. (L.) amazonensis) [25]; and (ii) yeasts and dermatophytes - D. hispida and C. suberosus $[25,42]$.

S. crassifolia and S. elliptica root wood extracts exhibited the strongest cytotoxicity, previously reported for the Salacia genus [36]. Another root extract, Simarouba versicolor root bark ethanol, presented strong activity against both osteosarcoma cell lines (Table 1). A previous study involving a hexane extract of the same $S$. versicolor organ led to the isolation of glaucarubinone, a cytotoxic compound [44].

From molecular networking analysis, S. crassifolia and S. elliptica extracts, as shown in the attached clusters formed, represent a different metabolic profile from the other plants, with metabolites not reported in the database (Figure S3, green highlighted circle). Furthermore, they exhibited the most potent activity against cancer cell lines in the highthroughput screening. C. sylvestris var. lingua and Cupania vernalis (Sapindaceae) extracts generated clusters which indicated similar metabolic profiles despite belonging to different plant families. However, C. sylvestris var. lingua exhibited more potent activity against 6 cancer cell lines ( $\mathrm{IC}_{50}$ value determination) and in the NCI-60 screen. The latter also showed higher activity against SF-295, HCT-8, MDA-MB-435 and LH-60 cell lines with $\mathrm{IC}_{50}$ values ranging from 0.5 and $2.1 \mu \mathrm{g} / \mathrm{mL}$ when compared to $C$. vernalis [44] 
An interesting result was noted regarding S. versicolor and D. hispida. Both species showed considerable activity, but did not form an exclusive cluster (noted in Figure S3). However, a few compounds were more intense (peak area intensity) in these plants than in the other species. A similar observation was made for Rapanea guianensis and Connarus suberosus (Connaraceae), which have comparable metabolite profiles. These observations pave the way for future studies to fractionate the extract and determine if a single active compound or combination of compounds results in the observed activity. For example, in this study, C. sylvestris var. lingua and C. vernalis are particularly interesting in that they presented high activity in the NCI-60 screen and showed 2 interesting cluster formations (Figures S2 and S3, red highlighted circle), with no compounds annotated. Further investigations involving isolation and characterization are necessary to elucidate the active compounds.

In conclusion, molecular networking provides a useful addition to the decision-making process when presented with of a large number of positive results. This streamlined strategy can not only economize time and resources by avoiding the isolation of known compounds, it also guides the isolation of unreported active compounds. Limitations of this analytical technique include the lack of compounds in databases and the possible presence of minor compounds that could account for the activity. Nevertheless, we have shown that a large amount of chemical information can be extracted using this strategy to provide an overview of chemical diversity and biological potential. We obtained metabolite information that did not match any compound described in the databases, possibly responsible for the cancer activity results. Finally, the chemical diversity from Brazilian Cerrado plants against cancer cell lines supports conservation of this biome hotspot to safeguard its valuable and unique biodiversity.

Supplementary Materials: The following are available online at https:/ / www.mdpi.com/2073-440 9/10/3/691/s1, Table S1. Brazilian Cerrado plant extracts submitted to high-throughput screening in a set of 8 cancer cell lines: colon (Colo205 and Km12), renal (A498 and U031), liver (HEP3B and SKHEP) and osteosarcoma (MG63 and MG63.3). Table S2. Z-factors for plates used in initial screening and secondary dose response testing. Figure S1. Complete molecular networking (MN) of 17 Cerrado plant extracts assayed in NCI-60. Figure S2. Expansion of the network, top portion. Figure S3. Expansion of the network, central portion. Figure S4. Expansion of the network, bottom portion. Figure S5-S55. Results graphs of NCI-60 screening.

Author Contributions: Conceptualization, L.S.E.; methodology, P.C.C., D.P.D., R.G.D., L.C.A., and L.S.E.; formal analysis, P.C.C., D.P.D., R.B.-F., E.I.G., and L.S.E.; investigation, P.C.C., R.G.D., L.C.A., and L.S.E.; resources, H.R.B,, K.R.G., J.A.B., and L.S.E.; writing-original draft preparation, P.C.C., D.P.D., R.G.D., L.C.A., R.B.-F., and L.S.E.; writing-review and editing, E.I.G., H.R.B,, K.R.G., J.A.B., and L.S.E.; supervision, H.R.B., K.R.G., J.A.B., and L.S.E.; project administration, H.R.B., K.R.G., J.A.B., and L.S.E.; funding acquisition, K.R.G., J.A.B., and L.S.E. All authors have read and agreed to the published version of the manuscript.

Funding: We wish to thank the Brazilian Scientific Research Agency CNPq-Process 249213/2013-3 and the special fund for the ArboControl Brazil Project, Ministry of Health-Process TED 74/2016 \& TED 42/2017 for financial support and fellowships.

Institutional Review Board Statement: Not applicable.

Informed Consent Statement: Not applicable.

Data Availability Statement: The data presented in this study are available on request from the corresponding author.

Acknowledgments: This work is dedicated to the memory of Professor José Elias de Paula, who was crucial for the collection, identification of the species, and construction of our extract bank.

Conflicts of Interest: The authors declare no conflict of interest. 


\section{References}

1. Newman, D.J.; Cragg, G.M. Natural Products as Sources of New Drugs over the Nearly Four Decades from 01/1981 to 09/2019. J. Nat. Prod. 2020, 83, 770-803. [CrossRef]

2. Lahsen, M.; Mercedes, M.C.; Bustamante, M.M.C.; Dalla-Nora, E.L. Undervaluing and Overexploiting the Brazilian Cerrado at Our Peril. Environ. Sci. Policy Sustain. Dev. 2016, 58, 4-15. [CrossRef]

3. Wang, M.X.; Carver, J.J.; Phelan, V.V.; Sanchez, L.M.; Garg, N.; Peng, Y.; Nguyen, D.D.; Watrous, J.; Kapono, C.A.; Luzzatto-Knaan, T.; et al. Sharing and Community Curation of Mass Spectrometry Data with Global Natural Products Social Molecular Networking. Nat. Biotechn. 2016, 34, 828-837. [CrossRef] [PubMed]

4. $\quad$ Pilon, A.C.; Selegato, D.M.; Fernandes, R.P.; Bueno, P.C.P.; Pinho, D.R.; Neto, F.C.; Freire, R.T.; Castro-Gamboa, I.; Bolzani, V.S.; Lopes, N.P. Metabolômica de Plantas: Métodos e Desafios. Quim. Nova 2020, 43, 329-354. [CrossRef]

5. $\quad$ Demarque, D.P.; Dusi, R.G.; de Sousa, F.D.M.; Grossi, S.M.; Silverio, M.R.S.; Lopes, N.P.; Espindola, L.S. Mass Spectrometry-Based Metabolomics Approach in the Isolation of Bioactive Natural Products. Sci. Rep. 2020, 10, 1051. [CrossRef] [PubMed]

6. Tosun, F.; Mihoglugil, F.; Beutler, J.A.A.; Eroglu Ozkan, E.; Miski, M. Neopapillarine, an Unusual Coumarino-Alkaloid from the Root Extract of Neocryptodiscus papillaris with Cytotoxic Activity on Renal Cancer Cells. Molecules 2020, $25,3040$. [CrossRef] [PubMed]

7. Devkota, K.P.; Covell, D.; Ransom, T.; McMahon, J.B.; Beutler, J.A. Growth Inhibition of Human Colon Carcinoma Cells by Sesquiterpenoids and Tetralones of Zygogynum Calothyrsum. J. Nat. Prod. 2013, 76, 710-714. [CrossRef]

8. Long, S.A.; Huang, S.; Kambala, A.; Ren, L.; Wilson, J.; Goetz, M.; Hao, X.; Yang, X.; Goncharova, E.I.; Jia, L.; et al. Identification of Potential Modulators Of Osteosarcoma Metastasis by High-Throughput Cellular Screening of Natural Products. Chem. Biol. Drug Des. 2021, 97, 77-86. [CrossRef]

9. Henrich, C.J.; Budhu, A.; Yu, Z.; Evans, J.R.; Goncharova, E.I.; Ransom, T.T.; Wang, X.W.; McMahon, J.B. High-Throughput Screening for Identification of Inhibitors of EpCAM-Dependent Growth of Hepatocellular Carcinoma Cells. Chem. Biol. Drug Des. 2013, 82, 131-139. [CrossRef] [PubMed]

10. Shoemaker, R.H. The NCI60 Human Tumour Cell Line Anticancer Drug Screen. Nat. Rev. Cancer 2006, 6, 813-823. [CrossRef]

11. Su, G.; Burant, C.F.; Beecher, C.W.; Athey, B.D.; Meng, F. Integrated Metabolome and Transcriptome Analysis of the NCI60 Dataset. BMC Bioinform. 2011, 12, 36. [CrossRef]

12. Holbeck, S.L.; Collins, J.M.; Doroshow, J.H. Analysis of Food and Drug Administration-Approved Anticancer Agents in the NCI60 Panel of Human Tumor Cell Lines. Mol. Cancer Ther 2010, 9, 1451-1460. [CrossRef] [PubMed]

13. EMBRAPA. Empresa Brasileira de Pesquisa Agropecuária. Mapa de Solos do Distrito Federal (1:100.000); EMBRAPA-PI. Serviço de Produção de Informação: Brasília, Brazil, 1978.

14. United States. Science, E Education Administration. Soil taxonomy: A Basic System of Soil Classification for Making and Interpreting Soil Surveys, 2nd ed.; US Department of Agriculture: Beltsville, MD, USA, 1975.

15. Tang, H.F.; Yun, J.; Lin, H.W.; Chen, X.L.; Wang, X.J.; Cheng, G. Two New Triterpenoid Saponins Cytotoxic to Human Glioblastoma U251MG Cells from Ardisia Pusilla. Chem. Biodivers. 2009, 6, 1443-1452. [CrossRef]

16. Dong, W.; Liu, X.; Li, X.; Yang, D.; Ding, L. A New Triterpene Saponin. Andr. Integr. Fitoter. 2011, 82, 782-785. [CrossRef]

17. Katajamaa, M.; Miettinen, J.; Oresic, M. MZmine: Toolbox for Processing and Visualization of Mass Spectrometry Based Molecular Profile Data. Bioinformatics 2006, 22, 634-636. [CrossRef]

18. Olivon, F.; Grelier, G.; Roussi, F.; Litaudon, M.; Touboul, D. MZmine 2 Data-Preprocessing to Enhance Molecular Networking Reliability. Anal. Chem. 2017, 89, 7836-7840. [CrossRef] [PubMed]

19. Myers, O.D.; Sumner, S.J.; Li, S.; Barnes, S.; Du, X. One Step Forward for Reducing False Positive and False Negative Compound Identifications from Mass Spectrometry Metabolomics Data: New Algorithms for Constructing Extracted Ion Chromatograms and Detecting Chromatographic Peaks. Anal. Chem. 2017, 89, 8696-8703. [CrossRef]

20. Nothias, L.F.; Petras, D.; Schmid, R.; Duhrkop, K.; Rainer, J.; Sarvepalli, A.; Protsyuk, I.; Ernst, M.; Tsugawa, H.; Fleischauer, M.; et al. Feature-Based Molecular Networking in the GNPS Analysis Environment. Nat. Methods 2020, 17, 905-908. [CrossRef]

21. Shannon, P.; Markiel, A.; Ozier, O.; Baliga, N.S.; Wang, J.T.; Ramage, D.; Amin, N.; Schwikowski, B.; Ideker, T. Cytoscape: A Software Environment for Integrated Models of Biomolecular Interaction Networks. Genome Res. 2003, 13, 2498-2504. [CrossRef] [PubMed]

22. Dictionary of Natural Products 28.1. Available online: http:/ / dnp.chemnetbase.com/ (accessed on 9 January 2020).

23. Tautenhahn, R.; Patti, G.J.; Rinehart, D.; Siuzdak, G. XCMS Online: A Web-Based Platform to Process Untargeted Metabolomic Data. Anal. Chem. 2012, 84, 5035-5039. [CrossRef]

24. Guijas, C.; Montenegro-Burke, J.R.; Domingo-Almenara, X.; Palermo, A.; Warth, B.; Hermann, G.; Koellensperger, G.; Huan, T.; Uritboonthai, W.; Aisporna, A.E.; et al. METLIN: A Technology Platform for Identifying Knowns and Unknowns. Anal. Chem. 2018, 90, 3156-3164. [CrossRef] [PubMed]

25. da Costa, R.C.; Santana, D.B.; Araujo, R.M.; de Paula, J.E.; do Nascimento, P.C.; Lopes, N.P.; Braz-Filho, R.; Espindola, L.S. Discovery of the Rapanone and Suberonone Mixture as a Motif for Leishmanicidal and Antifungal Applications. Bioorg. Med. Chem. 2014, 22, 135-140. [CrossRef] 
26. Zheng, Z.; Zhao, X.E.; Zhu, S.; Dang, J.; Qiao, X.; Qiu, Z.; Tao, Y. Simultaneous Determination of Oleanolic Acid and Ursolic Acid by in Vivo Microdialysis via UHPLC-MS/MS Using Magnetic Dispersive Solid Phase Extraction Coupling with MicrowaveAssisted Derivatization and Its Application to a Pharmacokinetic Study of Arctiumlappa, L. Root Extract in Rats. J. Agric. Food Chem. 2018, 66, 3975-3982. [CrossRef]

27. Amara, S.; Zheng, M.; Tiriveedhi, V. Oleanolic Acid Inhibits High Salt-Induced Exaggeration of Warburg-like Metabolism in Breast Cancer Cells. Cell Biochem. Biophys 2016, 74, 427-434. [CrossRef]

28. Ayeleso, T.B.; Matumba, M.G.; Mukwevho, E. Oleanolic Acid and Its Derivatives: Biological Activities and Therapeutic Potential in Chronic Diseases. Molecules 2017, 22, 1915. [CrossRef]

29. Novotny, L.; Abdel-Hamid, M.E.; Hamza, H.; Masterova, I.; Grancai, D. Development of LC-MS Method for Determination of Ursolic Acid: Application to the Analysis of Ursolic Acid in Staphylea holocarpa Hemsl. J. Pharm. Biomed. Anal. 2003, 31, 961-968. [CrossRef]

30. Kahnt, M.; Heller, L.; Grabandt, P.; Al-Harrasi, A.; Csuk, R. Platanic acid: A New Scaffold for the Synthesis of Cytotoxic Agents. Eur. J. Med. Chem. 2018, 143, 259-265. [CrossRef] [PubMed]

31. Lunardi, I.; Peixoto, J.L.B.; Silva, C.C.D.; Shuquel, I.T.A.; Basso, E.A.; Vidotti, G.J. Triterpenic Acids from Eugenia Moraviana. J. Braz. Chem. Soc. 2001, 12, 180-183. [CrossRef]

32. Fujioka, T.; Kashiwada, Y.; Kilkuskie, R.E.; Cosentino, L.M.; Ballas, L.M.; Jiang, J.B.; Janzen, W.P.; Chen, I.S.; Lee, K.H. Anti-AIDS Agents, 11. Betulinic Acid and Platanic Acid as Anti-HIV Principles from Syzigium Claviflorum, and the Anti-HIV Activity of Structurally Related Triterpenoids. J. Nat. Prod. 1994, 57, 243-247. [CrossRef]

33. Dhar Dubey, K.K.; Sharma, G.; Kumar, A. Conjugated Linolenic Acids: Implication in Cancer. J. Agric. Food Chem. 2019, 67, 6091-6101. [CrossRef]

34. Giordano, C.; Plastina, P.; Barone, I.; Catalano, S.; Bonofiglio, D. N-3 Polyunsaturated Fatty Acid Amides: New Avenues in the Prevention and Treatment of Breast Cancer. Int. J. Mol. Sci. 2020, 21, 2279. [CrossRef] [PubMed]

35. Okubo, R.; Noguchi, H.; Hamazaki, K.; Sekiguchi, M.; Kinoshita, T.; Katsumata, N.; Narisawa, T.; Uezono, Y.; Xiao, J.; Matsuoka, Y.J. Fear of Cancer Recurrence Among Breast Cancer Survivors Could be Controlled by Prudent Dietary Modification with Polyunsaturated Fatty Acids. J. Affect. Disord 2019, 245, 1114-1118. [CrossRef] [PubMed]

36. Espindola, L.S.; Dusi, R.G.; Demarque, D.P.; Braz, R.; Yan, P.C.; Bokesch, H.R.; Gustafson, K.R.; Beutler, J.A. Cytotoxic Triterpenes from Salacia Crassifolia and Metabolite Profiling of Celastraceae Species. Molecules 2018, 23, 1494. [CrossRef] [PubMed]

37. Zhao, Q.; Liu, Y.; Zhong, J.; Bi, Y.; Liu, Y.; Ren, Z.; Li, X.; Jia, J.; Yu, M.; Yu, X. Pristimerin Induces Apoptosis and Autophagy via Activation of ROS/ASK1/JNK Pathway in Human Breast Cancer in Vitro and in Vivo. Cell Death Discov. 2019, 5, 125. [CrossRef]

38. Kucera, O.; Mezera, V.; Moravcova, A.; Endlicher, R.; Lotkova, H.; Drahota, Z.; Cervinkova, Z. In Vitro Toxicity of Epigallocatechin Gallate in Rat Liver Mitochondria and Hepatocytes. Oxid Med. Cell Longev. 2015, 2015, 476180. [CrossRef] [PubMed]

39. Kurbitz, C.; Heise, D.; Redmer, T.; Goumas, F.; Arlt, A.; Lemke, J.; Rimbach, G.; Kalthoff, H.; Trauzold, A. Epicatechin Gallate and Catechin Gallate are Superior to Epigallocatechin Gallate in Growth Suppression and Anti-Inflammatory Activities in Pancreatic Tumor Cells. Cancer Sci. 2011, 102, 728-734. [CrossRef] [PubMed]

40. Bader, A.; Braca, A.; De Tommasi, N.; Morelli, I. Further Constituents from Caralluma Negevensis. Phytochemistry 2003, 62, 1277-1281. [CrossRef]

41. Wishart, D.S. Is Cancer a Genetic Disease or a Metabolic Disease? EBioMedicine 2015, 2, 478-479. [CrossRef] [PubMed]

42. Albernaz, L.C.; de Paula, J.E.; Romero, G.A.; Silva Mdo, R.; Grellier, P.; Mambu, L.; Espindola, L.S. Investigation of Plant Extracts in Traditional Medicine of the Brazilian Cerrado Against Protozoans and Yeasts. J. Ethnopharmacol 2010, 131, 116-121. [CrossRef]

43. De Mesquita, M.L.; Leao, W.F.; Ferreira, M.R.; de Paula, J.E.; Espindola, L.S.; Soares, L.A. Reversed-Phase-Liquid Chromatography Method for Separation and Quantification of Gallic Acid from Hydroalcoholic Extracts of Qualea Grandiflora and Qualea Parviflora. Pharmacogn. Mag. 2015, 11, 316-321. [CrossRef]

44. De Mesquita, M.L.; de Paula, J.E.; Pessoa, C.; de Moraes, M.O.; Costa-Lotufo, L.V.; Grougnet, R.; Michel, S.; Tillequin, F.; Espindola, L.S. Cytotoxic Activity of Brazilian Cerrado Plants Used in Traditional Medicine Against Cancer Cell Lines. J. Ethnopharmaco. 2009, 123, 439-445. [CrossRef] [PubMed] 